\title{
Analyses and Solid Waste Processing applications Construction Sand for construction of houses with high quality standards
}

\author{
Willy Hagi Teles Rego ${ }^{1}$, Adriano Lima Pedrosa ${ }^{1}$, Manoel S. Santos Azevedo ${ }^{1,2,3}$, Rivanildo Duarte Almeida ${ }^{3}$ \\ ${ }^{1}$ Universidade do Estado do Amazonas- Amazonas - Brasil. (hagi.willy@gmail.com) \\ ${ }^{2}$ Instituto Movimento Planeta - Amazonas - Brasil. (adrianolp01@gmail.com) \\ ${ }^{3}$ Itegam. - Amazonas - Brasil. (mazevedo@uea.edu.br)
}

\begin{abstract}
This paper analyzes the transformation of solid waste from rubbish to build houses with high quality standards. The analysis notes the effectiveness of technological innovation in civil construction, given the need to search for a new form of construction in which the current is outdated. Thus, several studies and sectors of science suggest a negative ecological impact as a result of this episode due to both the emissions to the atmosphere as the disposal of solid and liquid materials in nature, as well as gas emission to the atmosphere from potteries which are the manufacturers of conventional bricks. This paper presents analysis of the material that will be used in new technology, developed by the Institute Planet motion, which uses as starting material precisely these residues for the manufacture of a powder or sand transform which is later redestinations for various products have already been developed, such as artifacts for the construction of high standard houses, produced without any emission of gases into the atmosphere, a process that does not harm the environment and can be a great source of income.
\end{abstract}

Keywords: garbage; new technologies; sustainability.

\section{Análises e aplicações de Transformação de Resíduos sólidos da Construção Civil em Areia para a Construção de casas com alto padrão de qualidade}

\section{RESUMO}

Este artigo faz analises da transformação dos resíduos sólidos provenientes de entulhos para a construção de casas com alto padrão de qualidade. A análise constata a eficácia da inovação tecnológica na Construção civil, haja vista, a necessidade da busca de uma nova forma de construção em que a atual está já está ultrapassada. Assim, diversas pesquisas e setores da ciência apontam um impacto ecológico negativo como consequência deste episódio por conta tanto da emissão de poluentes para a atmosfera quanto o descarte de materiais sólidos e líquidos na natureza, bem como, emissão de gás para a atmosfera proveniente de olarias que são as fabricantes dos tijolos convencionais. Neste trabalho é apresentada análises do material que será utilizada na nova tecnologia, desenvolvida pelo Instituto Movimento Planeta, que usa como matéria prima justamente estes resíduos para a fabricação de um pó ou areia transformada que mais tarde é redestinada para diversos produtos, já desenvolvidos, como artefatos para a construção de casas de alto padrão de qualidade, produzida sem qualquer emissão de gases para a atmosfera, processo que não agride o meio-ambiente e pode ser uma grande fonte de renda.

Palavras Chaves: lixo; novas tecnologias; sustentabilidade.

\section{INTRODUÇÃO}

A Revolução Industrial iniciou o processo de mecanização e otimização dos meios de produção. Fábricas passaram a substituir a lenta atividade de manufatura existente por métodos mais rápidos e precisos neste episódio que é considerado por historiadores como o mais importante acontecimento social de todos. Com este acontecimento, bens eram criados em ritmo acelerado e tornaram-se mais acessíveis para a população comum, que migrava em peso das regiões rurais para os grandes centros urbanos de países como Inglaterra e França em busca de oportunidades de emprego e bem-estar. Como consequência deste chamado Exxodo Rural as cidades industrializadas foram crescendo cada vez mais e junto com a densidade demográfica.

A população, que passou a consumir mais, também despejava o lixo que produzia. Desorganizadamente na natureza

ITEGAM - JETIA Vol.02, Nº 06. Junho de 2016. Manaus - Amazonas, Brasil. ISSN 2447-0228 (ONLINE). 
causando diferenças cada vez mais significativas no clima e na biodiversidade do local.

Alterações físicas e ambientais causam modificações, muitas vezes negativa, nos ecossistemas [1]. A influência antrópica, i.e., a influência das atuações humanas no meioambiente, é cada vez mais forte por conta do consume desenfreado nas grandes cidades tanto por conta da população comum quanto pelas indústrias. [2] considera o crescimento urbano como a grande fonte de impactos ambientais das últimas décadas.

Uma forma de avaliar o impacto de cada indivíduo no meio-ambiente pode ser través da chamada "pegada de carbono"(carbon footprint), que mede a quantidade de dióxido de carbono que se produz diariamente e como estas emissões influenciam a natureza ao seu redor. Em média cada cidadão do mundo produz cerca de 4 toneladas deste gás por ano, sendo que o planeta atualmente comporta mais de 7 bilhões de pessoas. Nos continentes mais ricos, como na América do Norte, um cidadão comum produz em média até quatro vezes mais que a média global, ou seja, mais de 20 toneladas por ano! Como a pegada de carbono é uma medida do nosso cotidiano (o que comemos, a energia que gastamos, os meios de transporte que utilizamos etc), a forma de reduzi-la é justamente optar por alternativas na forma de que se vive e se consume.

No Brasil a tendência de abandono das áreas rurais em favor das regiões urbanas também se faz presente há quase um século, impulsionada por várias campanhas de industrialização desde os anos de Getúlio Vargas. Em média, o lixo doméstico no país, segundo [3], 65\% de matéria orgânica, 25\% de papel, $4 \%$ de metal, $3 \%$ de vidro e $3 \%$ de plástico. De todo este lixo, em apenas $33 \%$ dos municípios do Brasil (segundo uma pesquisa do IBGE feita no ano 2000) há uma coleta organizada do material descartado, enquanto na maior parte do país a população sofre sem os serviços mais básicos de saúde pública no que diz respeito ao recolhimento de lixo, que ainda acaba por retornar para a população durante eventos de enchentes.

No entanto, muito esforço é feito para a redução do impacto destes materiais no ambiente natural e, consequentemente, na vida das pessoas; da a reciclagem ao desenvolvimento de novas tecnologias mais sustentáveis. $\mathrm{O}$ presente trabalho visa introduzir uma alternativa para a destinação de materiais sólidos e líquidos e uma nova proposta de material que pode ser utilizado por diversos setores produtivos.

As formas de tratamento e destinação dos resíduos sólidos consistem em:

a) Reciclagem: A transformação de materiais usados em novos produtos para o consumo. Esta necessidade foi sentida pelos seres humanos a partir do momento em que foram verificados os benefícios que este procedimento pode trazer para o planeta Terra.

b) Compostagem: Processo de origem biológica a partir de decomposição da matéria orgânica contida em restos de origem animal ou vegetal. Esse conjunto de medidas tem como resultado final um produto - o composto orgânico - que deve permitir sua aplicação no solo sem ocasionar riscos ao meio ambiente.

Aterro Sanitário: Uma área destinada ao descarte final de resíduos sólidos não aproveitados gerados pela sociedade.

Incineração: É a queima de lixo em fornos e usinas próprias. A incineração é bastante utilizada no Brasil para o tratamento de resíduos biológicos.

\section{REVISÃO BIBLIOGRÁFICA}

Akinori Ito no inicio do ano 2010 criou um equipamento para transformar plástico em petróleo deste as criações da tecnologia já foram vendidas para mais 80 países, segundo o projetista a maquina não emite gases poluentes para a atmosfera, pois as moléculas são quebradas e transformadas em água e carbono durante a conversão do plástico em óleo, não causado impacto ao meio ambiente [4].

A empresa britânica DPS Global, criou uma tecnologia inovadora na área de resíduos sólidos, o equipamento e capaz leva as matérias a temperaturas elevadas, sem a presença de oxigênio, os resíduos são transformados em cinzas as partículas emitidas não causa danos a atmosfera do planeta terra [5].

A Solum Ambiental uma empresa que desenvolver tecnologias inovadoras na área de tratamentos de resíduos sólidos .Criou uma tecnologia que usar um reator contendo fontes térmicas, a maquina conseguir dar destinação final a qualquer que seja o material solido [6].

\section{MATERIAIS E MÉTODOS}

Pesquisadores do Instituto Movimento Planeta [7], uma instituição de pesquisas e soluções para o tratamento e redestinação de resíduos sólidos e líquidos, começaram a desenvolver um projeto que pretende dar melhor tratamento aos problemas maléficos ao meio ambiente. Cada cidadão da Terra contribui com toneladas de dióxido de carbono anualmente, agredindo a natureza. Novas tecnologias podem e estão sendo desenvolvidas com um novo destino ao lixo que são produzidos. Através destes materiais, o desafio da equipe torna-se transformálos em uma outra forma de material que possa ser utilizado em vários setores produtivos, utilizando também os esforços de diversas engenharias.

Os resíduos sólidos, que comumente denomina-se de lixo, selecionado através de um sistema embarcado com capacidade de separar os resíduos de acordo com seu tipo, é triturado e segue para um processo que retira odores através de uma técnica não-divulgada pelos pesquisadores do Instituto Movimento Planeta. Por fim, este lixo triturado torna-se uma espécie de areia ou pó como é mostrado na figura 1, que pode ser utilizada como matéria prima para a construção civil, como vista 
na figura 2, substituindo materiais como o cimento e tijolos convencionais.

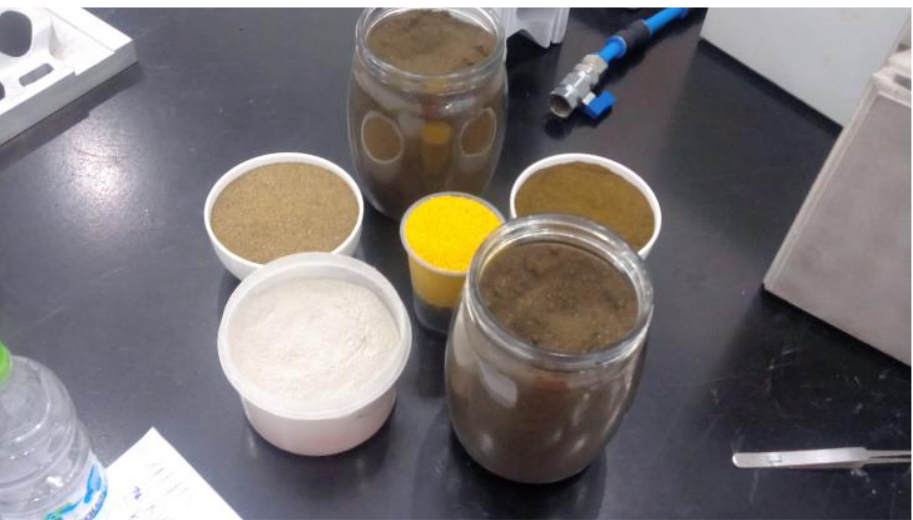

Figura 1 - Lixo tratado em forma de areia.

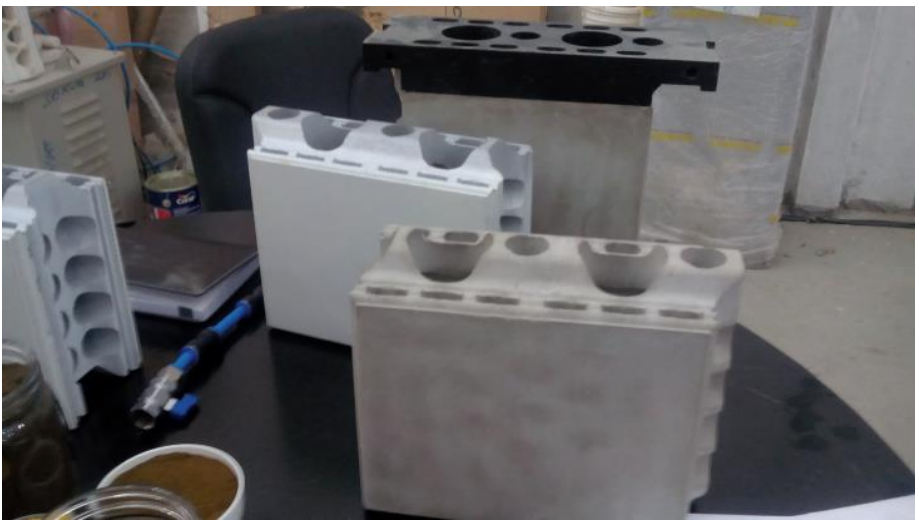

Figura 2 - Blocos construídos a partir da areia da Redestinação de resíduos sólidos da construção civil.

\section{EXPERIMENTOS: ÁNALISES PRELIMINARES}

O desenvolvimento experimental deu-se nos laboratórios do Instituto Movimento Planeta e tem por objetivo comprovar que a areia originada a partir da transformação de resíduos da construção civil, podem ser reaproveitados como agregado apropriado para produção de argamassa, em composição a princípio com elementos ligantes, tipo cimento convencional, desde que se observe alguns procedimentos necessários à sua utilização.

De acordo com [8], a viabilidade de utilização de um produto deve ser comprovada com análise das características que podem ser oferecidas por ele e que tenha condição de satisfazer as necessidades e exigências dos clientes. Considera-se que essas características por apresentarem possibilidades de mensuração relativas às quantidades exigidas, são as variáveis de resposta para o nosso estudo.

Para a análise desenvolvida de forma experimental destacam-se variáveis dependentes de resposta:

Massa especifica do concreto no estado fresco;

> Resistência à compressão do concreto;

$>$ Absorção;
$>$ Trabalhabilidade.

Definidas as variáveis dependentes, será necessário a definição das variáveis independentes, que segundo [4], são os parâmetros do processo.

\section{ANÁLISE GRANULOMÉTRICA}

A distribuição granulométrica da amostra tal qual (TQ, britada abaixo de $19 \mathrm{~mm}$ ), bem como da fração $<3 \mathrm{~mm}$ da amostra tal qual (TQ-areia), e da areia-VSI, são apresentadas comparativamente na Figura1.

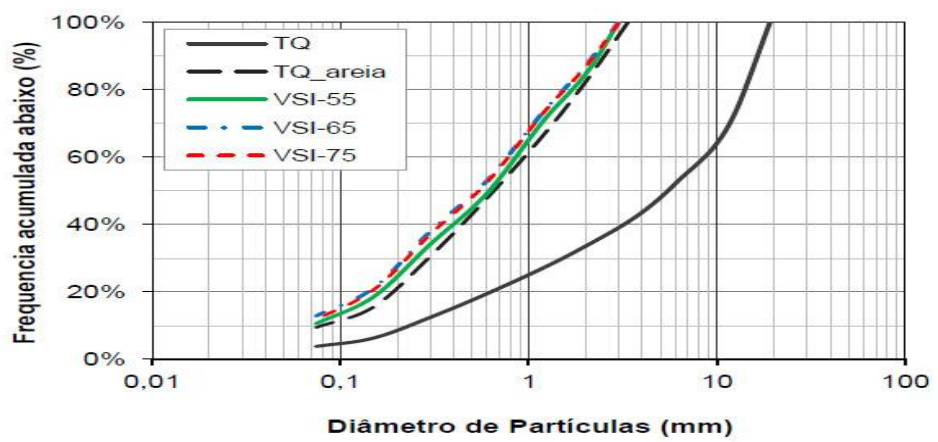

Figura 1 - Distribuições granulométricas comparativas

A distribuição granulométrica da amostra tal qual indica que a proporção de agregados graúdos (acima de $4,8 \mathrm{~mm}$ ) e miúdos (abaixo de 4,8 mm) é semelhante: $53 \%$ e $47 \%$, respectivamente, sendo que cerca de um quarto da amostra tem dimensão superior a $12,7 \mathrm{~mm}(25,7 \%)$. Por outro lado, a fração abaixo de $0,15 \mathrm{~mm}$ corresponde a $6,3 \%$ a massa total, sendo $3,9 \%$ abaixo de $0,074 \mathrm{~mm}$.

Considerando-se apenas a fração abaixo de $3 \mathrm{~mm}$ presente na amostra tal qual (denominada TQ-areia), verifica-se que $15 \%$ de sua massa está abaixo de $0,15 \mathrm{~mm}$.

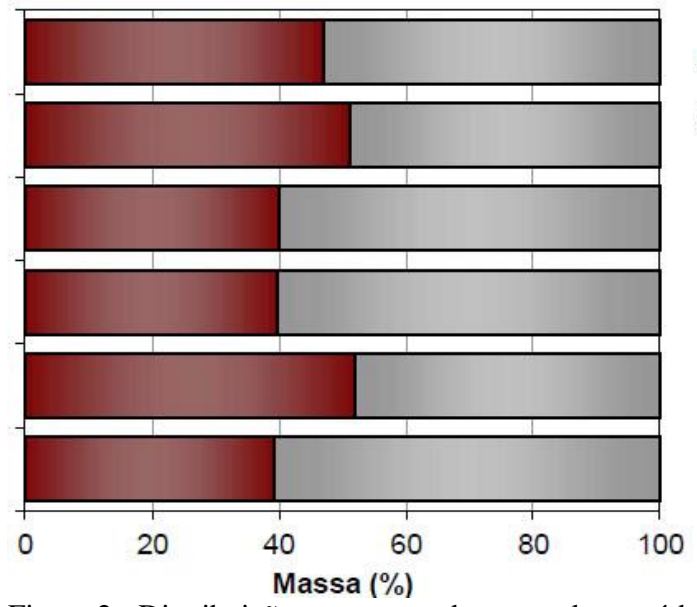

-Graúdo $>4,8 \mathrm{~mm}$ 口Miúdo $<4,8 \mathrm{~mm}$

Figura 2 - Distribuições em massa de agregados graúdos e miúdos [9].

A partição em massa de agregados graúdos e miúdos gerados na britagem do RCD na usina Urbem se assemelha a outros resíduos nacionais de outras localidades, conforme dados 
Willy Hagi Teles Rego, et al / ITEGAM-JETIA Vol.02, No 06, pp.28-32. Junho, 2016.

de estudo realizado por Ulsen, Kahn e colaboradores [9] ilustrados na Figura 4.2.

Os produtos gerados pelo VSI apresentam distribuições granulométricas muito semelhantes, especialmente nas velocidades de 65 e $75 \mathrm{~m} / \mathrm{s}$. O produto VSI-55 apresenta uma distribuição discretamente mais grossa.

Dos gráficos comparativos de distribuição granulométrica, nota-se claramente a semelhança nas curvas dos produtos TQ-areia e da areia-VSI. A proporção em massa de material abaixo de $0,15 \mathrm{~mm}$ aumentou de $15 \%$ para TQ-areia para $19 \%$ a $21 \%$ nas areias-VSI, ao passo que a proporção abaixo de $0,074 \mathrm{~mm}$ variou de $9 \%$ para 11 a $13 \%$, respectivamente. Os resultados demonstram que a britagem terciária reduziu a dimensão máxima característica da amostra de $19 \mathrm{~mm}$ para $3 \mathrm{~mm}$ com acréscimo de apenas 4 a $5 \%$ na proporção de finos, demonstrando a habilidade do VSI no controle sobre a granulometria do produto.

Os produtos gerados pelo VSI apresentam distribuições granulométricas muito semelhantes, especialmente nas velocidades de 65 e $75 \mathrm{~m} / \mathrm{s}$. O produto VSI-55 apresenta uma distribuição discretamente mais grossa.

Dos gráficos comparativos de distribuição granulométrica, nota-se claramente a semelhança nas curvas dos produtos TQ-areia e da areia-VSI. A proporção em massa de material abaixo de $0,15 \mathrm{~mm}$ aumentou de $15 \%$ para TQ-areia para $19 \%$ a $21 \%$ nas areias-VSI, ao passo que a proporção abaixo de $0,074 \mathrm{~mm}$ variou de $9 \%$ para 11 a $13 \%$, respectivamente. Os resultados demonstram que a britagem terciária reduziu a dimensão máxima característica da amostra de $19 \mathrm{~mm}$ para $3 \mathrm{~mm}$ com acréscimo de apenas 4 a $5 \%$ na proporção de finos, demonstrando a habilidade do VSI no controle sobre a granulometria do produto.

Dessa forma conclui-se que a britagem terciária abaixo de $3 \mathrm{~mm}$ gera um produto (areia-VSI) com distribuição granulométrica bastante semelhante à fração areia $(<3 \mathrm{~mm})$ presente no produto de britagem secundária abaixo de $19 \mathrm{~mm}$ (TQ-areia).

\section{CONCLUSÃO}

Pode-se observar então que o lixo produzido pela sociedade é o intuito de suprir nossas necessidades ao mesmo tempo em que reduzem o impacto na natureza, como a tecnologia apresentada neste trabalho. Esta tecnologia, por sua vez, pode ser aplicada em diversos ramos da construção civil, empregando também diversos setores das engenharias, sendo também um material sustentável criado a partir de resíduos sólidos e líquidos.

\section{AGRADECIMENTOS}

Instituto Movimento Planeta, a Universidade do Estado do Amazonas (UEA) e ao Instituto de Tecnologia e Educação Galileo da Amzônia (ITEGAM).

\section{REFERÊNCIAS BIBLIOGRÁFICAS}

[1] MUCELIN, C. A., Bellini, M. (2008). Lixo e impactos ambientais perceptíveis no ecossistema urbano. Sociedade natureza, 20(1), 111-124.

[2] ODUM, Eugene Pleasants Warrett, and Gary W. Aguilar Ortega. Fundamentos de ecologia. No. 574.5 O35. 2006.

[3] LEITE, M. B. Avaliação das propriedades mecânicas de concretos produzidos com agregados reciclados de resíduos de construção e demolição. 2001. 270f. Tese (Doutorado em Engenharia Civil), 2001.

[4] MANZINI, Ezio,VEZZOLI, Carlo. O desenvolvimento de produtos sustentáveis: os requisitos ambientais dos produtos industriais.São Paulo: Edusp, 2002.

[5] UOL, fonte- essas e outras disponível em::<http://essaseoutras.xpg.uol.com.br/transformacao-deplastico-em-petroleo-maquina-criada-por-akinori-ito/>. Acesso em 14 de maio de 2016.

[6]TNPETROLEO, http://tnpetroleo.com.br/noticia/tecnologiatrata-residuos-solidos-em-plataformas-e-embarcacoes/ Acesso em 14 de maio de 2016.

[7] AZEVEDO, Manoel S. S. Uma proposta para redestinação dos resíduos sólidos e líquidos, propondo também a criação do terceiro setor na cadeia produtiva industrial, 2016.

[8] LEITE, M. B.; CORDEIRO, L. N. P.; MASUEIRO, A. B.; DAL MOLIN, D. C. C. Proposta de adaptação do procedimento proposto por Leite (2001) para determinação da absorção de agregados reciclados de resíduos de construção e demolição. In CONGRESSO INTERNACIONAL SOBRE PATOLOGIA E REABILITAÇÃO DE ESTRUTURAS， $7^{\circ}$, 2011. Anais CINPAR, 2011.

[9] ULSEN, K. Caracterização tecnológica de resíduos de construção e demolição. São Paulo: Escola Politécnica, Universidade de São Paulo, 2010. 20p.

[10] GRIMBERG, E.; BLAUTH, P. Coleta Seletiva: reciclando materiais, reciclando valores. Polis: estudos, formação e assessoria em políticas sociais, n.31, 1998.

[11] JARDIM, N. S.; WELLS, C. (Org.). Lixo Municipal: Manual de Gerenciamento integrado. São Paulo: IPT: CEMPRE, 1995.

[12] NUSEC - Núcleo de Sócio Econômico da Universidade Federal do Amazonas. Estudo da cadeia produtiva de embalagens de papelão no pólo industrial de Manaus (PIM). Manaus: UFAM, 2009. 
